\title{
A Note Concerning the Reflection of Waves in Inhomoge- neous Layers With Asymmetric Profiles
}

\author{
R. Burman \\ Department of Physics, Victoria University of Wellington, New Zealand
}

(Received December 28, 1964)

\begin{abstract}
Approximate formulas for reflection coefficients have previously been used to study the reflection of waves in inhomogeneous layers having symmetric profiles of the refractive index or its gradient [Heading, 1963; Wait and Jackson, 1964; Burman, 1965]. In the present note the extension of this work to the case of asymmetric profiles is considered.
\end{abstract}

\section{Introduction}

The reflection coefficient $r$ for VHF radio waves incident on inhomogeneous layers which occur in the troposphere is given by the approximate formula [Wait, 1962; Wait and Jackson, 1964]

$$
r=\frac{1}{2 C^{2}} \int_{-\infty}^{\infty} \frac{d n}{d z} e^{-i 2 k C z} d z
$$

where $n(z)$ is the refractive index and $z$ is the height. Also, $C$ is the cosine of the angle of incidence of the waves and $k=\omega / c, \omega$ being the angular frequency and $c$ the speed of light in free space. A time factor $e^{i \omega t}$ has been assumed. This formula is applicable to both horizontally and vertically polarized waves [Wait, 1962].

Exact expressions for the integral in eq (1) can be obtained when the refractive index gradient $d n / d z$ is taken to have certain functional forms [Wait, 1962]. Of particular physical interest are cases in which $d n / d z$ is taken to be continuous everywhere. In the continuous profiles which have been studied [Wait and Jackson, 1964; Burman, 1965] the function $d n / d z$ possesses a single maximum or minimum and is symmetric about the position of the extreme value. The purpose of the present note is to give expressions for the reflection coefficient in the more general case in which $d n / d z$ is not symmetric. This will enable a wider range of models of the tropospheric layers to be dealt with. The results can also be applied to the study of the reflection of electromagnetic waves in an underdense plasma by using approximate formulas given by Heading [1963].

\section{An Asymmetric Profile}

In the present note the profile,

$$
\begin{aligned}
\frac{d n}{d z} & =\frac{2^{m} A_{m}}{\left(e^{a z}+e^{-b z}\right)^{m}} \\
& =\frac{A_{m} \exp \{m(b-a) z / 2\}}{\cosh ^{m}\{(a+b) z / 2\}},
\end{aligned}
$$

will be considered where $a$ and $b$ are taken to be real and positive and independent of $z$. Also, $m$ is a positive integer and $A_{m}$ is independent of $z$. When $b=a$, this profile reduces to a symmetric profile, as previously considered [W ait and Jackson, 1964; Burman, 1965]. When $b \neq a$, the profile is asymmetric.

It is found from tables of transforms [e.g., Campbell and Foster, 1948, p. 68] that

$$
\int_{-\infty}^{\infty} \frac{e^{p x} d x}{\cosh ^{m}(q x)}=\frac{2^{m-1}}{q \Gamma(m)} \Gamma\left(\frac{m}{2}+\frac{p}{2 q}\right) \Gamma\left(\frac{m}{2}-\frac{p}{2 q}\right)
$$

where $\Gamma$ represents the gamma function. Using this, (1) and (3) give

$$
r=\frac{2^{m-1} A_{m}}{(a+b) C^{2} \Gamma(m)} \Gamma\left(\frac{m}{2}+\alpha\right) \Gamma\left(\frac{m}{2}-\alpha\right),
$$

where

$$
\alpha=\frac{m(b-a)-i 4 k C}{2(a+b)} .
$$


Using the relations [Magnus and Oberhettinger, 1949, p. 1]

$$
\begin{aligned}
\Gamma(\nu) \Gamma(1-\nu) & =\pi \operatorname{cosec}(\nu \pi), \\
\Gamma\left(\frac{1}{2}+\nu\right) \Gamma\left(\frac{1}{2}-\nu\right) & =\pi \sec (\nu \pi), \\
\Gamma(\nu+1) & =\nu \Gamma(\nu)
\end{aligned}
$$

and

$$
\Gamma(1)=1, \quad \Gamma(2)=1,
$$

it is found that

$$
\frac{r_{1}}{A_{1}}=\frac{\pi}{(a+b) C^{2} \cos \left(\alpha_{1} \pi\right)}
$$

and

$$
\frac{r_{2}}{A_{2}}=\frac{2 \alpha_{2} \pi}{(a+b) C^{2} \sin \left(\alpha_{2} \pi\right)},
$$

where the subscripts 1 and 2 denote the cases $m=1$ and $m=2$, respectively. When $b \neq a$, the expressions (7) and (8) are both complex, but when $b=a$ they are real. Thus, introduction of the asymmetry causes a change in phase of the reflection coefficient as well as a change in its modulus.

If $x$ and $y$ are real, then

$$
|\cos (x+i y)|=\left(\cos ^{2} x+\sinh ^{2} y\right)^{1 / 2}
$$

and

$$
|\sin (x+i y)|=\left(\sin ^{2} x+\sinh ^{2} y\right)^{1 / 2} .
$$

Using these expressions, (7) and (8) give (with $a, b$ real and positive and $C$ real)

$$
\left|r_{1}\right|=\frac{\pi\left|A_{1}\right|}{(a+b) C^{2}}\left\{\cos ^{2}\left[\frac{\pi(b-a)}{2(a+b)}\right]+\sinh ^{2}\left[\frac{2 k C \pi}{a+b}\right]\right\}^{-1 / 2}
$$

and

$$
\begin{aligned}
\left|r_{2}\right|=\frac{2 \pi\left|A_{2}\right|}{(a+b)^{2} C^{2}}\left\{(b-a)^{2}+4 k^{2} C^{2}\right\}^{1 / 2} & \\
& \times\left\{\sin ^{2}\left[\frac{\pi(b-a)}{a+b}\right]+\sinh ^{2}\left[\frac{2 k C \pi}{a+b}\right]\right\}^{-1 / 2} .
\end{aligned}
$$

By differentiating (3) it is found that the layer has an extreme value at height $z=z_{0}$ where $z_{0}$ satisfies

$$
\tanh \left[\left(a+\frac{\delta}{2}\right) z_{0}\right]=\frac{\delta}{2 a+\delta}
$$

where $\delta \equiv b-a$. If $\delta$ is small the layer differs only slightly from the symmetric case. For small $\delta$ equation (13) gives

$$
z_{0} \doteqdot \delta /\left(2 a^{2}\right)
$$

which is valid for $\delta \ll 2 a$. In the symmetric case $\delta=0$ and the peak occurs at $z=0$.

From (3) and (14) it is found that to first order in $\delta$ the value of $d n / d z$ at the peak is $A_{m}$, as it is in the symmetric case. Thus to first order, the effect of a small $\delta$ on the peak of the profile is to shift it from its position for $\delta=0$ without altering the peak value.

When $\delta$ is small it is found that to first order in $\delta$,

$$
\begin{array}{r}
\left|r_{1}\right|=\frac{\pi\left|A_{1}\right|}{2 a C^{2} \cosh (k C \pi / a)}\left[1+\frac{\delta}{2 a}\left\{\left(\frac{k C \pi}{a}\right)\right.\right. \\
\left.\left.\tanh \left(\frac{k C \pi}{a}\right)-1\right\}\right]
\end{array}
$$

and

$$
\begin{aligned}
\left|r_{2}\right|=\frac{\pi k\left|A_{2}\right|}{a^{2} C \sinh (k C \pi / a)}\left[1+\frac{\delta}{a}\left\{\left(\frac{k C \pi}{2 a}\right)\right.\right. & \\
& \left.\left.\operatorname{coth}\left(\frac{k C \pi}{a}\right)-1\right\}\right] .
\end{aligned}
$$

If the slightly asymmetric layer were behaving as the corresponding symmetric layer with its peak displaced, then $r$ would differ from its value in the symmetric case only by the phase factor $e^{-i 2 k C z_{0}}$. That is, to first order in small $\delta,|r|$ would be unaffected by $\delta$. However, (15) and (16) show that, except when the expressions in curly brackets vanish, $\left|r_{1}\right|$ and $\left|r_{2}\right|$ depend on the first power of $\delta$ for small $\delta$. Even a slight asymmetry can have a first order effect on $|r|$. This is interpreted to mean that the shift in the peak is not the only significant effect of a slight asymmetry in the profile. The effect of the asymmetry in regions away from the peak may also be important.

Heading [1963] studied the reflection of electromagnetic waves in a stratified ionized medium by expressing the reflected field as an integral over contributions scattered back from elementary layers of thickness $\delta z$ situated at height $z$ in the medium. Expressions for the reflection coefficients were obtained by using the Born approximation, thus neglecting multiple scattering. The approximate reflection coefficients given by Heading [1963] for an isotropic layer can be written

$$
R_{h} \doteqdot \frac{i k}{2 C} \int_{-\infty}^{\infty}\left(1-n^{2}\right) e^{-i 2 k C z} d z
$$

and

$$
R_{v} \doteqdot \frac{i k\left(2 C^{2}-1\right)}{2 C} \int_{-\infty}^{\infty}\left(1-n^{2}\right) \mathrm{e}^{-i 2 k C z} d z
$$

where $R_{h}$ and $R_{v}$ are the reflection coefficients for horizontally and vertically polarized waves, respec- 
tively. In these equations the symbols have the same meanings as in (1).

The expressions (17) and (18) are similar to (1) except that the integrands are proportional to $\left(1-n^{2}\right)$ instead of $d n / d z$. Thus the profile given by (2) and (3) can be used to study the reflection of waves in ionized layers in which $\left(1-n^{2}\right)$, which is proportional to electron density, is a nonsymmetric function of height.

(Paper 69D5-505)

\section{References}

Burman, R. (March, 1965), The reflection of VHF waves from tropospheric layers with 'hyperbolic' profiles, IEEE Trans. Ant. Prop. AP-13, No. 2, 324

Campbell, G. A., and R. M. Foster (1948), Fourier Integrals for Practical Applications (D. Van Nostrand Co., New York).

Heading, J. (Jan-Feb, 1963), Composition of reflection and transmission formulae, J. Res. NBS 67D (Radio Prop.), No. 1, 65-77.

Magnus, W., and F. Oberhettinger (1949), Formulas and Theorems for the Special Functions of Mathematical Physics (Chelsea Publ. Co., New York).

Wait, J. R. (1962), Electromagnetic Waves in Stratified Media, pp. 93-95 (Pergamon Press, Oxford).

Wait, J. R. and C. M. Jackson (July, 1964), Influence of the refractive index profile in VHF reflection from a tropospheric layer, IEEE Trans. Ant. Prop. AP-12, No. 4, 512-513. 